\title{
Detecting Movement Type by Route Segmentation and Classification
}

\author{
Karol Waga, Andrei Tabarcea, Minjie Chen, Pasi Fränti \\ Speech \& Image Processing Unit, School of Computing \\ University of Eastern Finland, Joensuu \\ \{karol.waga, andrei.tabarcea, minjie.chen, pasi.franti\}@uef.fi
}

\begin{abstract}
Data about people movement is nowadays easy to collect by GPS technology embedded in smartphones. GPS routes provide information about position, time and speed, but further conclusion requires either prior information or data analysis. We propose a method to detect the movement type by segmentation of the GPS route using speed, direction and their derivatives, and by applying an inference algorithm via a second order Markov model. The method is able to classify most typical moving types such as motor vehicle, bicycle, run, walk and stop.
\end{abstract}

Keywords: route analysis, segmentation, classification, GPS trajectory routes, tracks, mobile applications, second order Markov model.

\section{Introduction and Motivation}

Mobile phones have developed rapidly and most people are using one nowadays. Many of the phones, especially smartphones, are equipped with multiple sensors including global positioning system (GPS). This makes it possible to implement various locationaware services varying from recommendation of pointof-interests [9] to sport activity tracking. GPS data, however, can capture only features such as speed, distance, location and time which cannot be used to conclude semantic meaning of the user activity [5].

In this work, we aim at providing higher-level information of the user activity by detecting five most typical movement types: stop, walk, run, bicycle, and motor vehicle. The proposed method is based on route segmentation and a simple rule-based classifier as follows.

First, segmentation is performed in order to divide the analyzed route into a number of segments, where the sum of the inner speed variance is minimized. A set of basic features, such as speed, acceleration, time, direction and distance are then extracted for each segment. In the second stage, the extracted segments are classified into a predefined set of five classes using a second order Markov model.

The proposed method is implemented and tested using the data collected by users of MOPSI (in Finnish MObiilit PaikkatietoSovellukset ja Internet, Mobile Location-based Application and Internet) services (see Fig. 1). It implements location-aware services such as user tracking, route recording, photo collection, recommendations, bus schedules and sharing data in Facebook. All these are available through website and mobile platforms in Symbian, Android, iPhone and Windows Phone devices. The proposed movement type detection algorithm includes three steps that are preprocessing, segmentation and classification. They are implemented in $\mathrm{C}$ language and executed on server real-time when user requests route analysis.

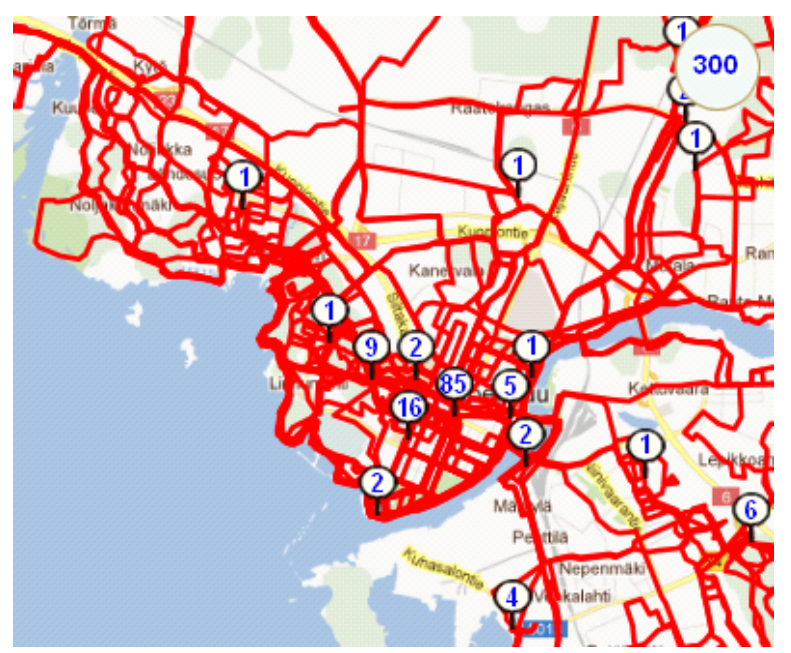

Figure 1. Sample data collected in MOPSI route system: http://cs.uef.fi/mopsi/ 
We demonstrate by qualitative tests that the proposed method can successfully detect most typical movement types based on raw GPS data only. Better accuracy would require the use of other sensors such as phone accelerometer, heart rate, or using external information such as road network, bus stops and bus schedules. Geographic information could be used for separating between land and water although not enough to separate boating from cross-country skiing on lake.

\section{Related work}

Most work on the automatic determination of travel mode uses GPS data, but few attempts exist to use other types of data including call detail records [10] and cellular network positioning data. A study on different combinations of GPS, GSM, WiFi and accelerometer was constructed in [8]. It concludes that the combination of GPS and accelerometer is the best data source for the travel model detection.

A simple approach is to measure the speed of the GPS device, which is then compared with empirical thresholds $[1,7]$. However, some transport modes, such as cycling and running, are hard to differentiate using only the speed thresholds. More complex solutions have therefore been considered, based on methods such as fuzzy logic [11], neural networks [4] and hidden Markov model [8, 13].

$\mathrm{Xu}$ et al. [11] proposed travel mode detection using fuzzy logic with five movement types: walk, bike, bus, rail and rest. The routes are divided into stages using fuzzy pattern recognition and a min-max operation. The variables used for fuzzy calculations are median speed, average speed, standard deviation of speed and minimum acceleration. Membership functions are created for each fuzzy variable and the travel mode is detected with satisfying accuracy, except rail.

Using neural networks in travel mode classification was proposed in [4]. Adaptive sampling rate (1s rate outdoor and lower rate indoor) and on-device route reduction called critical point algorithm are used to conserve energy. Classification uses the following attributes: average speed, maximum speed, estimated horizontal accuracy uncertainty, percentage of GSM fixes compared to GPS, standard deviation of distances between stop locations, average dwell time (how much does a stop take). The neural network uses just the critical points to classify a route as car, walk or bus and the accuracy of this method is $80-90 \%$.

A three-stage approach was proposed in [13] consisting of the following steps: segmentation by change point detection, feature selection followed by classification using an inference model and graphbased probabilistic post-processing. The advantages of the approach are that it can effectively segment routes with various transportation modes, it is dependent only on GPS data (no additional sensor data or map information) and the model learned from some users can be applied to infer GPS data from other users. However, in most of the cases, the change points are considered to be walk points. Therefore, only nonmoving or walking parts of the trajectory are detected as segment boundaries. Additionally, we consider speed changes as segment boundaries, having different segmentation results, for instance when the user travels from highway to urban areas by car.

In [8], a decision tree followed by a first-order hidden Markov model is used. This method has $93.6 \%$ accuracy and it does not rely on GIS information or historical data as in [13]. The paper also compares different classifiers: decision trees, k-means clustering, naïve Bayes, nearest neighbor, support vector machines, continuous hidden Markov model, and a decision tree combined with a discrete hidden Markov model. The decision tree classifier provided the most accurate result of the simple classifiers, and its combination with discrete hidden Markov model was the most accurate overall.

These have two main weaknesses that we address. Firstly, training material is not always available, and may result in over-fitting, which loses generalization. Secondly, the correlation between neighboring segments is not fully exploited and depends on the segmentation accuracy. By using second-order Markov model we address this problem and we benefit from the correlation to the previous and also to the next segment.

\section{Proposed Solution}

The challenge of the movement type detection is demonstrated in Fig. 2 and Fig. 3.

The first example in Fig. 2 includes interval training with three faster segments separated by shorts stops, and two slower jogging periods in the beginning and in the end. The challenge here is to accurately detect the stop points. The labels in these pictures are ground truth provided by the person who performed the exercise.

The second example in Fig. 3 includes three fast downhill skiing segments, two slow uphill movements by elevator, and two waiting periods (lining up in queue waiting to access the skiing elevator). Although it is not possible to conclude all this activity merely 
from the GPS data, segmentation can help to obtain better classification.

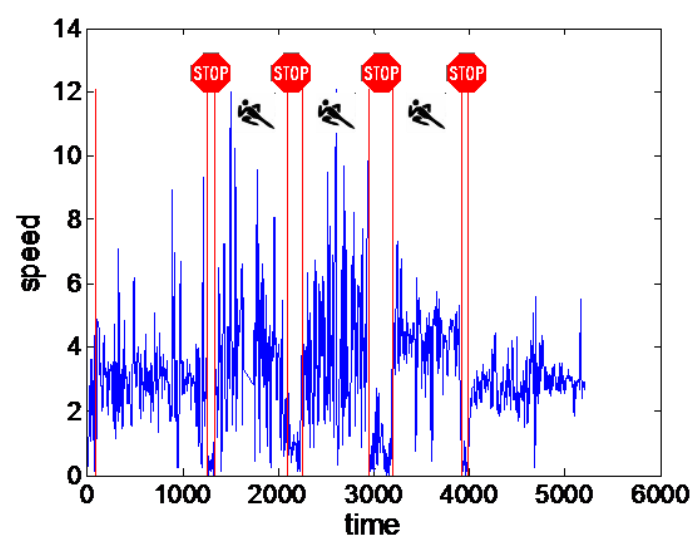

Figure 2. Non-trivial example of movement type analysis: interval training.

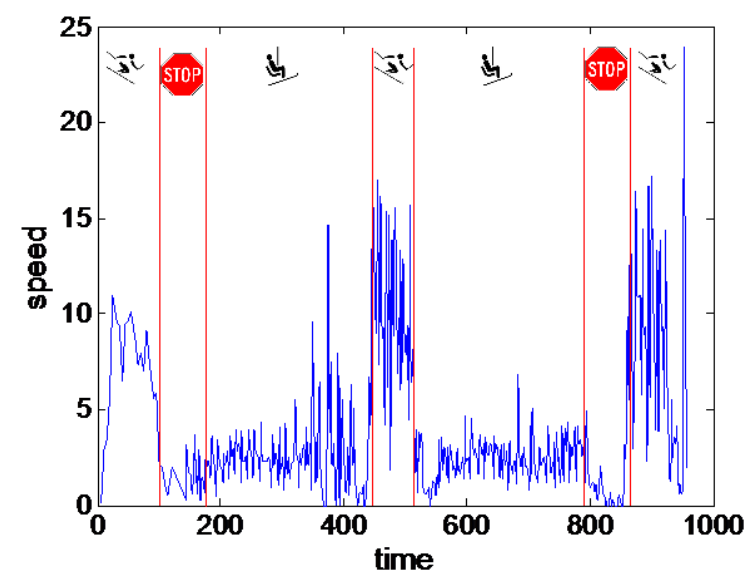

Figure 3. Non-trivial example of movement type analysis: quality skiing time in elevator.

\section{A. Route Segmentation}

We first divide a route into segments with similar speed. The number of segments is automatically determined but could also be predefined by user.

An input to the algorithm is a route $P=\left(p_{1}, p_{2}, \ldots, p_{n}\right)$, where $p_{i}=\left(x_{i}, y_{i}, t_{i}\right)$, and the corresponding speeds are $\left(v_{1}, v_{2}, \ldots, v_{n-1}\right)$. For a given segment number $m$, we define a cost function that minimizes the sum of the inner speed variance in all the segments:

$$
f=\sum_{j} \sigma_{i_{j}}^{i_{j+1}}\left(t_{i_{j+1}}-t_{i_{j}}\right)
$$

where $i_{j}$ and $i_{j+1}$ are the indexes of the start and end points of the segment $j$, and $\sigma$ is the speed variance between the points $j$ to $j+1$ in route $p_{\text {i }}$. Our experiments have shown that the proposed cost function is more efficient than mean square error, which has difficulties to detect walking segments with lower speed.

This minimization process is solved by a dynamic programming process in $\mathrm{O}\left(n^{2} m\right)$ time and $\mathrm{O}(n m)$ space, where the speed variance can be calculated in $\mathrm{O}(1)$ time by using the pre-calculated accumulated sums. Optimization is done in the $n \times m$ state space using dynamic programming as follows:

$$
\begin{aligned}
& D(s, r)=\min \left(D(c, r-1)+\sigma_{c}^{s}\left(t_{s}-t_{c}\right)\right), c \in(1 \ldots s-1) \\
& A(s, r)=\arg \min _{c}\left(D(c, r-1)+\sigma_{c}^{s}\left(t_{s}-t_{c}\right)\right)
\end{aligned}
$$

where $s=0 \ldots n, r=0 \ldots m$, with an initial condition $D(0$, $0)=0$, and $A(s, r)$ is the index for backtracking. The number of segments $m_{0}$ is determined by

$$
m_{0}=\arg \min _{i}\left(D(n, i)+\lambda_{1} i+\lambda_{2}\left(t_{n}-t_{1}\right)\right), i=1 \ldots m
$$

where $\lambda_{1}, \lambda_{2}$ are regularization parameters.

\section{B. Moving Type Classification}

Several features such as speed, acceleration, time, direction and distance can be calculated. However, training a classifier directly on these features is not accurate, since many features overlapped over different movement types [13]. Instead, we first perform soft classification of each segment as stop, walk, run, bicycle or motor vehicle using the a priori probabilities shown in Fig. 4.

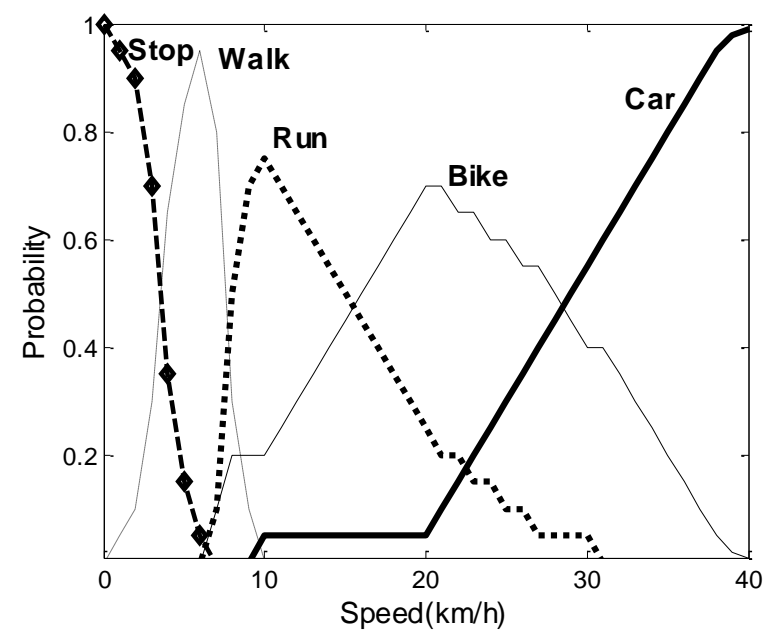

Figure 4. A priori probabilities for soft classification of the route segments.

On the other hand, the first order hidden Markov model (HMM) has been successfully used to exploit correlations between neighboring segments [8]. In this model, the hidden states represent the movement types and the observed data are the features for each segment. We extend this to a second-order HMM by 
exploiting correlations both to the previous and the next segment.

The state transition matrix is empirically initialized as shown in Table 1, but it could also be optimized via a training process in further stages of the application if training data exists.

Table 1: Transition probabilities used in the 2-HMM

\begin{tabular}{|c|c|c|c|c|c|c|}
\hline \multirow[b]{2}{*}{ Prev. } & \multicolumn{5}{|c|}{ Probability: } & \multirow[b]{2}{*}{ Next } \\
\hline & 응 & $\$$ & 安 & $\dot{k}$ & (5100) & \\
\hline 응 & 0.6 & - & - & 0.2 & 0.2 & 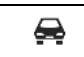 \\
\hline$\Leftrightarrow$ & 0.5 & 0.2 & - & 0.1 & 0.2 & ठठ \\
\hline$\Leftrightarrow$ & 0.5 & - & 0.2 & 0.1 & 0.2 & 实 \\
\hline$\Leftrightarrow$ & 0.5 & - & - & 0.3 & 0.2 & $\dot{k}$ \\
\hline 응 & 0.8 & - & - & 0.1 & 0.1 & अ100 \\
\hline హె & 0.5 & 0.2 & - & 0.1 & 0.2 & $\Leftrightarrow$ \\
\hline$\$$ & - & 0.6 & _ & 0.2 & 0.2 & ळ \\
\hline ळ & - & 0.4 & 0.4 & 0.1 & 0.1 & 实 \\
\hline$\$$ & - & 0.4 & - & 0.4 & 0.2 & $\dot{k}$ \\
\hline$\$$ & - & 0.8 & - & 0.1 & 0.1 & (अ०ि) \\
\hline 安 & 0.5 & - & 0.2 & 0.1 & 0.2 & $\Leftrightarrow$ \\
\hline 实 & - & 0.4 & 0.4 & 0.1 & 0.1 & ठ \\
\hline 定 & - & - & 0.4 & 0.4 & 0.2 & 安 \\
\hline 安 & - & - & 0.4 & 0.4 & 0.2 & $\dot{k}$ \\
\hline 实 & - & - & 0.8 & 0.1 & 0.1 & (100) \\
\hline$\dot{k}$ & 0.5 & - & - & 0.3 & 0.2 & $\Leftrightarrow$ \\
\hline$\dot{k}$ & - & 0.4 & - & 0.4 & 0.2 & ळర \\
\hline$\dot{R}$ & - & - & 0.4 & 0.4 & 0.2 & 务 \\
\hline$\dot{k}$ & 0.2 & 0.2 & 0.2 & 0.2 & 0.2 & $\dot{k}$ \\
\hline$\dot{R}$ & - & - & 0.1 & 0.7 & 0.2 & (अ10) \\
\hline अ100 & 0.8 & - & - & 0.1 & 0.1 & 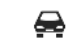 \\
\hline (अ100 & - & 0.8 & - & 0.1 & 0.1 & ळర \\
\hline (1900) & - & - & 0.8 & 0.1 & 0.1 & 安 \\
\hline (अ100) & - & - & 0.1 & 0.7 & 0.2 & $\dot{k}$ \\
\hline (अ०) & 0.2 & 0.2 & 0.2 & 0.2 & 0.2 & (10) \\
\hline
\end{tabular}

For the cost function of the $2^{\text {nd }}$ order HMM we use:

$$
f=\prod_{i=1}^{M} P\left(m_{i} \mid \mathrm{X}_{\mathrm{i}}, m_{i-1}, m_{i+1}\right)
$$

where $m_{i}=\{$ stop, walk, run, bicycle or motor vehicle $\}$ is the state of segment $i, \mathrm{X}_{\mathrm{i}}$ is its feature vector, $m_{i-1}$, $m_{i+1}$ are the states of the previous and the next segment. The probability that a segment would have a hidden state $m_{i}$ depends on the previous state, the next state and its feature vector. After Eq. (4) has been maximized, the most likely sequence of the hidden state $m_{0}, m_{1} \ldots m_{M}$ is determined.

Assuming the feature vector $\mathrm{X}_{\mathrm{i}}$ is uncorrelated with $m_{i-1}$ and $m_{i+1}$, this cost function can be converted by applying Bayesian inference:

$$
f=\prod_{i=1}^{M} \frac{P\left(m_{i+2} \mid m_{i}, m_{i+1}\right) P\left(m_{i+1} \mid X\right)}{P\left(m_{i+2}\right)}
$$

where $P\left(m_{i+2} \mid m_{i}, m_{i+1}\right), P\left(m_{i} \mid \mathrm{X}_{\mathrm{i}}\right)$ and $P\left(m_{i}\right)$ are all prior information. In the implementation of the algorithm, dynamic programming is employed for maximizing the cost function (5). The maximizing is done in a similar manner as Viterbi algorithm, which has been used for the first order HMM.

\section{Experiments}

The proposed system is implemented in Mopsi, which is publicly available (http://cs.uef.fi/mopsi). Segmentation and travel mode classification are triggered via Analyze function in the routes view. After analysis, the statistics of the segments (speed, distance and movement type) are displayed. In Mopsi, there are more than 7000 routes that in total have more than 4 million points, which are selected here as qualitative examples to demonstrate the capability and limitations of the algorithm.

Fig. 5 shows a car tour with one stop at traffic lights. The speed segments demonstrate typical traffic flow in Joensuu. The last segment is classified also as motor vehicle based on the overall pattern despite its slower speed.

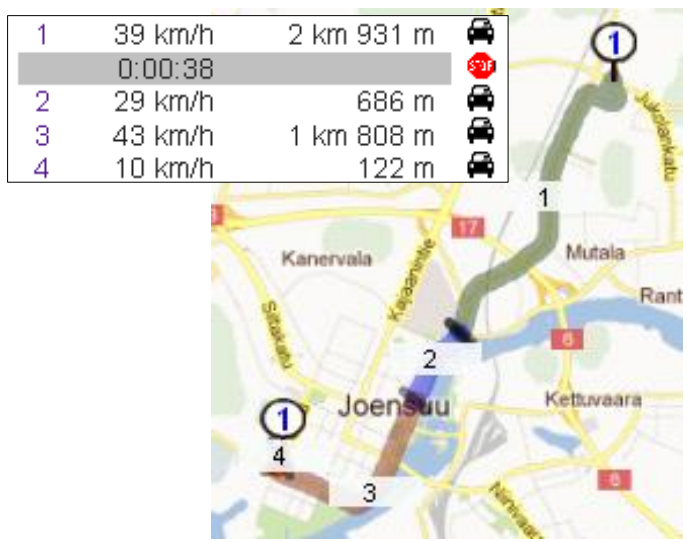

Figure 5. Segmentation of a car route with one stop.

Fig. 6 and 7 show sport exercises where the running speed can be easily concluded from the moving segments, even though one short stop (segment 2) is incorrectly classified as walking. 


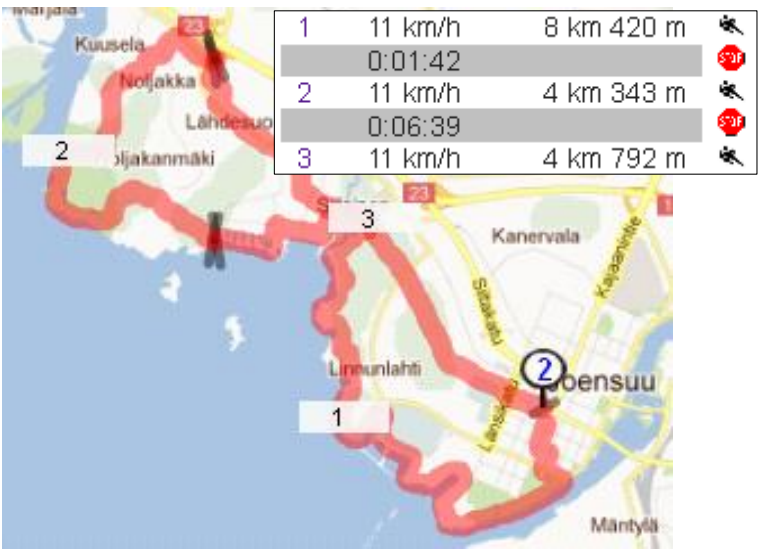

Figure 6. Separating stop segments from running.

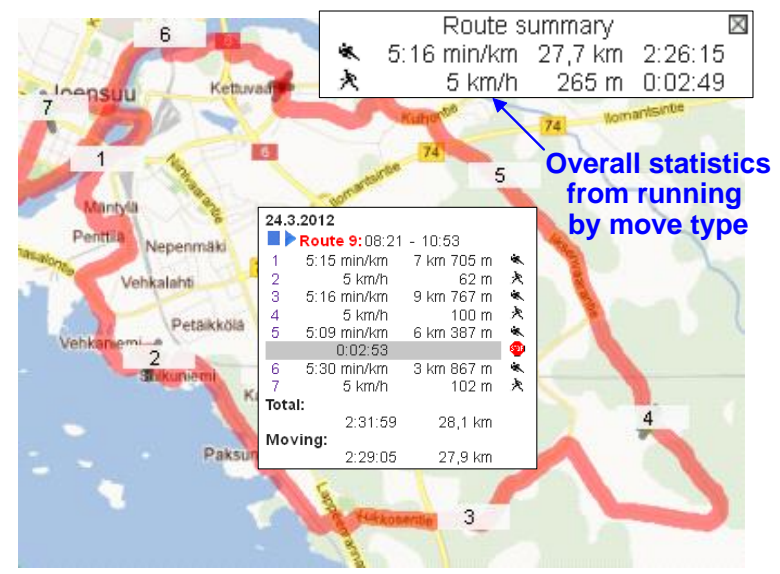

Figure 7. Long-distance running.

In Fig. 8, three interval exercises are captured, along with warm-up and slow-down. The classifier recognizes the exercises correctly as running segments with similar average speed $(13 \mathrm{~km} / \mathrm{h})$. The segmentation itself makes the analysis also easier for the user.

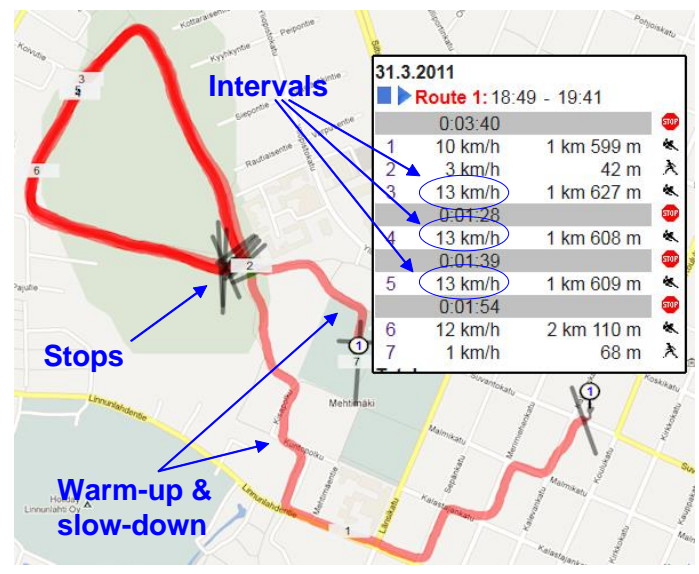

Figure 8. Interval training exercise
The cycling segments in the route in Fig. 9 are incorrectly classified as motor vehicle. Despite the accurate segmentation and the correct detection of walking segment, the inaccuracies of GPS signal and high top speed cause incorrect classification. One segment classified as motor vehicle movement increases the probability of similar segments to be classified with the same transportation mode.

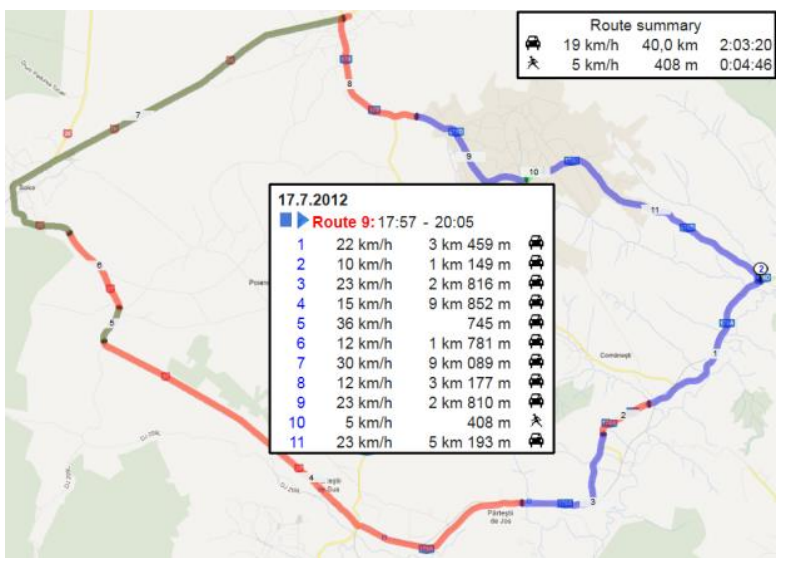

Figure 9. Bicycle route classified as car.

Finally, Fig. 10 shows that our segmentation algorithm can detect speed changes as segment boundaries, even though the transportation mode is the same (car),

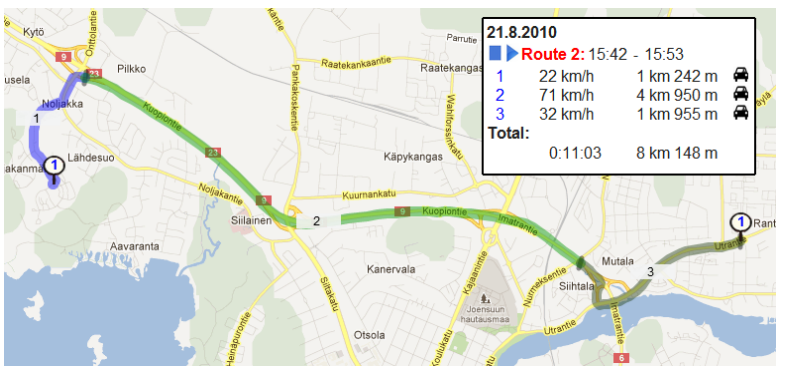

Figure 10. Travelling by car on highway between urban areas.

\section{Discussion and Conclusions}

The proposed approach was demonstrated to be useful for analyzing collected GPS trajectories and detecting typical movement types such as motor vehicle, bicycle, run, walk and stop. In some cases, segmentation missed short stops, and separation between bicycle and running would require userspecific information or data from accelerometer.

As a future work, we plan to implement annotation tool using the model in [5] to collect ground truth and perform numerical comparison with other methods. 


\section{References}

[1] Bohte, W., Maat, K. Deriving and validating trip purposes and travel modes for multi-days GPS-based travel surveys: A largescale application in the Netherlands. Transport Research, Part C 17, p. 285-297. 2009.

[2] Chen, M., Xu, M., Fränti, P. Compression of GPS Trajectories. Data Compression Conference. Snowbird, USA, 62-71, April 2012.

[3] Fränti, P., Tabarcea, A., Kuittinen, J., Hautamäki, V. Locationbased search engine for multimedia phones. IEEE Int. Conf. on Multimedia \& Expo (ICME'10), 558-563, Singapore, July 2010.

[4] Gonzalez, P.A., Weinstein, J.S., Barbeau, S.J., Labrador, M.A., Winters, P.L., Georggi, N.L., Perez, R. Automating Mode Detection for Travel Behaviour Analysis by Using Global Postioning Systems-enabled Mobile Phones and Neural Networks. IET Intelligent Transportation Systems, 4 (1), 37 49. 2010.

[5] Guc, B. Semantic Annotation of GPS Trajectories. International Conference on Geographic Information Science. Park City, Utah, USA, September 2008.

[6] Lee, W.-Ch., Krumm, J. Chapter 1: Trajectory Preprocessing, in Computing with Spatial Trajectories, Springer, 2011
[7] Oliveira, M., Troped, P. J., Wolf, J., Matthews, C. E., Cromley, E. K., Melly, S. J. Mode and Activity Identification Using GPS and Accelerometer Data. Transportation Research Record, 2006.

[8] Reddy, S., Mun, M., Burke, J., Estrin, D., Hansen, M. Srivastava, M. Using Mobile Phones to Determine Transportation Modes. ACM Transactions on Sensor Networks, 6 (2), 1-27, 2010.

[9] Waga, K., Tabarcea, A., Fränti, P. Context Aware Recommendation of Location-based Data. Int. Conf. on System Theory, Control and Computing. Sinaia, Romania, October 2011.

[10] Wang, H., Calabrese, F., Di Lorenzo, G., Ratti, C. Transportation Mode Inference from Anonymized and Aggregated Mobile Phone Call Detail Records. Int. Conf. on Intelligent Transportation Systems, Madeira, Portugal, September 2010.

[11] Xu, Ch., Ji, M., Chen, W., Zhang, Z. Identifying Travel Mode from GPS Trajectories through Fuzzy Pattern Recognition. Int Conf. on Fuzzy Systems and Knowledge Discovery, Yantai, China, August 2011.

[13] Zheng, Y., Chen, Y., Li, Q., Xie, X., Ma, W.-Y. Understanding Transportation Modes Based on GPS Data for Web Applications, ACM Trans. on Web, 4 (1), 2010. 